ESAIM: PROCEEDINGS AND SURVEYS, September 2018, Vol. 62, p. 79-90 Muhammad DAUHOO, Laurent DUMAS, Pierre GABRIEL and Pauline LAFITTE

\title{
THIN STRUCTURES WITH IMPOSED METRIC
}

\author{
Marta LewiCKA ${ }^{1}$ And Annie RaOulT ${ }^{2}$
}

\begin{abstract}
We consider thin structures with a non necessarily realizable imposed metric, that only depends on the surface variable. We give a unified presentation of the three main limit models. We establish the generalized membrane model and we show, by means of an algebraic proof, that the internal membrane energy vanishes on short maps of the metric restricted to the plane. We recall that a generalized bending model can occur only when this reduced metric admits sufficiently regular isometric immersions. When the entries $R_{12}$.. of the Riemannian curvature tensor are null, this bending energy can vanish; then the next model is necessarily a generalized von Kármán model whose minimum is zero if and only if the three-dimensional metric is flat.
\end{abstract}

Résumé. Nous considérons des structures fines à métrique imposée, dépendant des variables planaires, non nécessairement réalisable. Nous donnons une présentation synthétique des trois principaux modèles limites. Nous établissons un modèle de membrane généralisé et montrons que son énergie interne s'annule pour les déformations non expansives de la métrique restreinte au plan. Nous rappelons qu'un modèle de flexion généralisé ne peut apparaître que si cette métrique réduite admet des immersions isométriques suffisamment régulières, et que, dès lors que les composantes $R_{12}$.. du tenseur de courbure sont nulles, l'énergie de flexion peut s'annuler. Le modèle suivant est alors nécessairement un modèle de von Kármán généralisé dont le minimum est nul si et seulement si le tenseur de courbure est nul, c'est-à-dire si la métrique de départ est plate.

\section{INTRODUCTION}

Obtaining models for slender structures has been a long-standing issue. In the 50s, first attempts to derive lower-dimensional models from three-dimensional systems consisted in simply cancelling terms expected to be small for a slender structure. Then, researchers settled the topic in an asymptotic framework were convergence results may be obtained. This point of view allowed to see several models as part of a hierarchy depending on the magnitude of the applied loads or, equivalently, on the internal energy. This hierarchy was extensively studied [9], [11] for three-dimensional elastic structures that, when unloaded, are at rest in a reference configuration. Most recently, experimental setups that allow to reproduce the effects of a prestrain on the shape of thin films were designed. In [13], [14], thin gel films undergo nonuniform shrinkage when activated in a hot bath according to a prescribed radially symmetric prestrain. Large-scale buckling, multi-scale wrinkling or symmetrybreaking patterns appear in the sheets, depending on the nature of the "programmed in" metrics, see Figure 1. Controlling of shape through prestrain was proposed as well in [23], where temperature-responsive gel sheets that can transform between a flat state and a prescribed three-dimensional shape are obtained by photopatterning polymer films, see Figure 2. For other experimental results see [12,21,24].

\footnotetext{
${ }^{1}$ University of Pittsburgh, Department of Mathematics, 301 Thackeray Hall, Pittsburgh, PA 15260, USA

${ }^{2}$ Laboratoire MAP5, Université Paris Descartes \& CNRS, Sorbonne Paris Cité, France
}

(C) EDP Sciences, SMAI 2018 


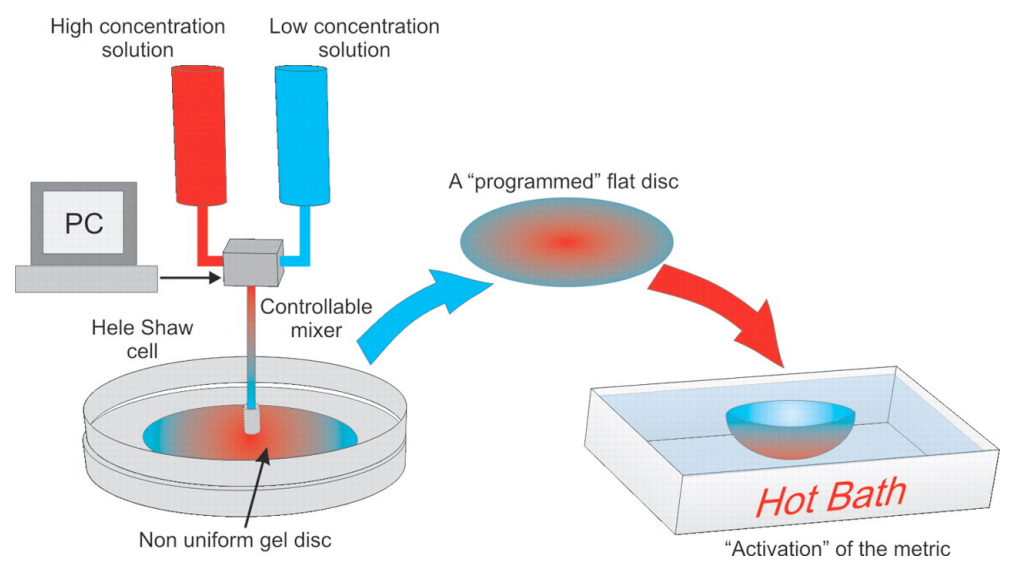

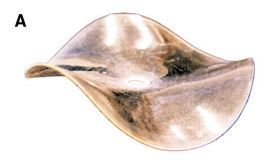

B

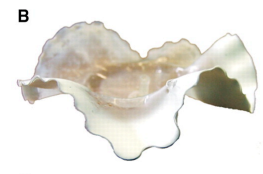

c

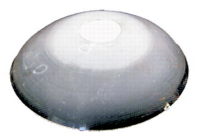

D

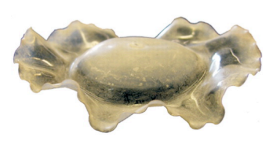

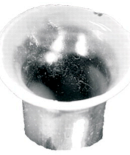

$\mathrm{F}$

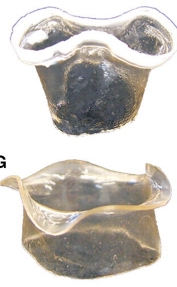

H

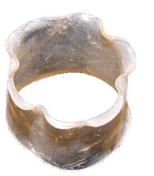

FIGURE 1. Manufacturing thin sheets with radially symmetric target metrics [13]. Reprinted with permission from AAAS.
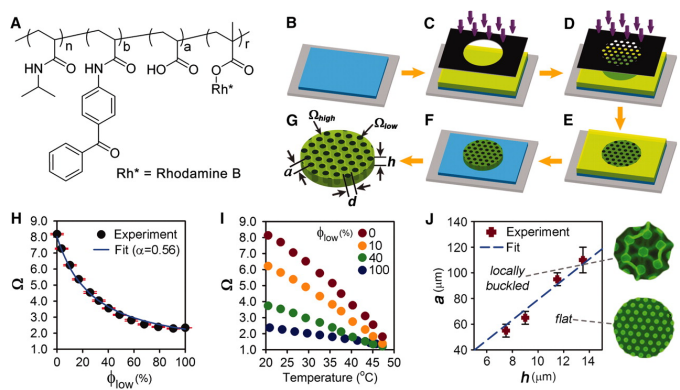
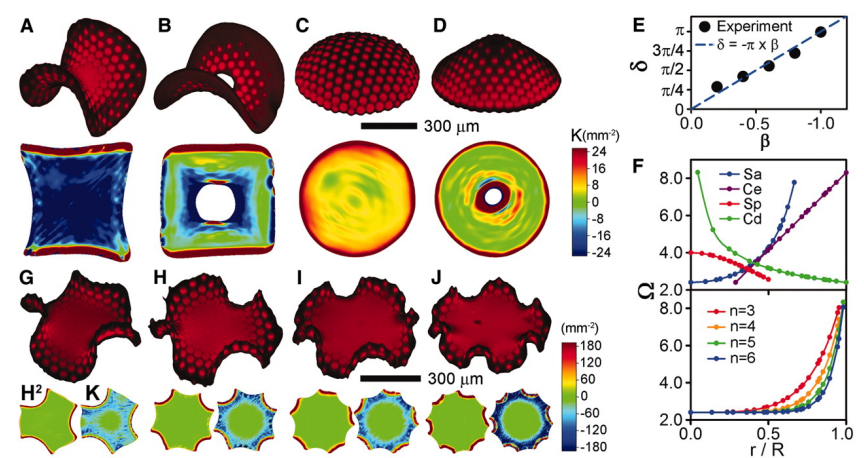

Figure 2. Halftone gel lithography [23]. Reprinted with permission from AAAS.

In this short survey, we concentrate on three main models that are well understood, namely the membrane model, the bending model, the von Kármán model and their appropriate generalizations in the non Euclidean setting. We underline the fact that the structure of the Riemann curvature tensor of the target metric plays a significant role in identifying the limit internal energy and determining whether it can vanish, a feature which is not seen in the classical Euclidean setting.

\section{Membrane model and Short maPS}

In this section, we first settle the problem: given three-dimensional slender elastic structures, we aim at identifying limit models when the thickness goes to 0 . We work in the context where, at the 3d-level, an unloaded structure aims at reaching an orientation-preserving configuration $\phi: O \mapsto \mathbb{R}^{3}$ such that $(\nabla \phi)^{T} \nabla \phi=G$ where a symmetric matrix field $G$ is given. This may be not realizable, i.e. the exact solution to the mentioned system of PDEs may not exist. We identify the first limit model and discuss its interest. In particular, we give a short algebraic proof of the vanishing of the membrane energy on short maps. 
Let be given an open set $O \subset \mathbb{R}^{3}$, the reference configuration of a body, and a target metric $G: O \mapsto$ Sym $_{3}^{>}$ where $\mathrm{Sym}_{3}^{>}$is the space of positive definite $3 \times 3$ symmetric matrices. We denote by $A$ the positive definite, symmetric, square root of $G$.

Our modeling hypothesis is as follows, see [8], [18], [3]: submitted to external loads $f$, the three-dimensional nonlinear elastic body with space-dependent target metric $G$ seeks to mimimize the total energy

$$
I(\psi)=E(\psi)-\int_{O} f(x) \cdot \psi(x) \mathrm{d} x:=\int_{O} W\left(x, \nabla \psi(x) A^{-1}(x)\right) \mathrm{d} x-\int_{O} f(x) \cdot \psi(x) \mathrm{d} x,
$$

over deformations $\psi: O \mapsto \mathbb{R}^{3}$ that may be required to satisfy a boundary condition of place. We assume that $W \geq 0, W$ is frame-indifferent, $W(x, R)=0$ for any $x \in O, R \in \mathrm{SO}(3)$. Then, the internal energy vanishes if $(\nabla \psi)^{T} \nabla \psi=G$, det $\nabla \psi(x)>0$, which is consistent with the modeling of a target metric. The dependency on the space variable $x$ appears twice in the stored energy density $W$, thus allowing to deal with bodies that may have at two separate points the same target metric but non equal mechanical constants. We assume $G$ to be smooth. Indeed, the several behaviors we will describe here are related to the geometrical structure of $G$, so that a possible lack of regularity is not under focus. Under the assumption that $O$ is simply connected, the fundamental theorem of Riemannian geometry asserts that $G$ admits an isometric immersion $\psi: O \mapsto \mathbb{R}^{3}(i . e$. $(\nabla \psi)^{T} \nabla \psi=G$, det $\left.\nabla \psi(x)>0\right)$ defined in the weakest possible way, that is in $H^{1}\left(O ; \mathbb{R}^{3}\right)$, if and only if the Riemannian curvature tensor $\mathcal{R}$ of $G$ vanishes identically. In addition, the immersion is automatically smooth. A stronger result stated in Proposition 1.1 below is true. This result was originally obtained in [18] in the spirit of the quantitative rigid estimate [10]. Using Ball's arguments, we give a new proof.

Proposition 1.1. If the Riemannian curvature tensor of the metric $G$ is not zero, then

$$
\inf \left\{\left\|\operatorname{dist}\left(\nabla \psi(\cdot) A^{-1}(\cdot), \operatorname{SO}(3)\right)\right\|_{\left.L^{2}(O)\right)} ; \psi \in H^{1}\left(O ; \mathbb{R}^{3}\right)\right\}>0
$$

Proof. We argue by contradiction. Suppose that there exists a sequence $\psi_{n}$ in $H^{1}\left(O ; \mathbb{R}^{3}\right)$ such that, when $n$ goes to $+\infty$,

$$
\left\|\operatorname{dist}\left(\nabla \psi_{n}(\cdot) A^{-1}(\cdot), \mathrm{SO}(3)\right)\right\|_{L^{2}(O)} \rightarrow 0 .
$$

Classically, see [10], we can assume without loss of generality that for some $M,\left\|\nabla \psi_{n}\right\|_{L^{\infty}\left(O ; \mathbb{M}_{3,3}\right)} \leq M$ for all $n$, which implies $\left\|\operatorname{Cof}\left(\nabla \psi_{n}\right)\right\|_{L^{\infty}\left(O ; \mathbb{M}_{3,3}\right)} \leq M^{\prime}$ for some $M^{\prime}$. Then, there exists $\psi \in H^{1}\left(O ; \mathbb{R}^{3}\right), H \in L^{2}\left(O ; \mathbb{M}_{3,3}\right)$, such that, for a subsequence, $\nabla \psi_{n} \rightarrow \nabla \psi$ and $\operatorname{Cof}\left(\nabla \psi_{n}\right) \rightarrow H$ in $L^{2}\left(O ; \mathbb{M}_{3,3}\right)$. By Ball's arguments, see [1], $H=\operatorname{Cof}(\nabla \psi)$. Now, by [10] again, there exists $C>0$ such that for all $F,|F| \leq M$, one has $|F-\operatorname{Cof} F| \leq$ $C \operatorname{dist}(F, \mathrm{SO}(3))$. Therefore,

$$
\left\|\nabla \psi_{n} A^{-1}-\operatorname{Cof}\left(\nabla \psi_{n} A^{-1}\right)\right\|_{L^{2}\left(O ; \mathbb{M}_{3,3}\right)} \rightarrow 0
$$

from which we deduce, using $\operatorname{Cof}\left(\nabla \psi_{n} A^{-1}\right)=\operatorname{Cof}\left(\nabla \psi_{n}\right) \operatorname{Cof}\left(A^{-1}\right) \rightarrow \operatorname{Cof}(\nabla \psi) \operatorname{Cof}\left(A^{-1}\right)$,

$$
\nabla \psi A^{-1}=\operatorname{Cof}\left(\nabla \psi A^{-1}\right)
$$

On the one hand, (4) implies that $\operatorname{Cof}(\nabla \psi)=(\operatorname{det} G)^{1 / 2}(\nabla \psi) G^{-1}$ which, since $\operatorname{div} \operatorname{Cof}(\nabla \psi)=0$, provides $\Delta_{G} \psi=0$ where $\Delta_{G}$ is the Laplace-Beltrami operator with respect to $G$. Then, $\psi \in C^{\infty}\left(O ; \mathbb{R}^{3}\right)$. On the other hand, one can check that for a given matrix $F$ in $\mathbb{M}_{3,3}$, the identity $F=$ Cof $F$ is equivalent to $F=0$ or $F \in \mathrm{SO}(3)$. Since $\nabla \psi$ is continuous, (4) implies that either $\psi$ is constant or $\nabla \psi A^{-1}(x) \in \operatorname{SO}(3)$ for all $x$. In the latter case, the fundamental theorem of Riemannian geometry imposes that $\mathcal{R}=0$ and the contradiction is obtained. It remains to exclude the former case. Coming back to (2),

$$
\exists R_{n} \in L^{2}(O ; \mathrm{SO}(3)),\left\|\nabla \psi_{n}(\cdot) A^{-1}(\cdot)-R_{n}(\cdot)\right\|_{L^{2}\left(O ; \mathbb{R}^{3}\right)} \rightarrow 0
$$

Then, for a subsequence, $R_{n}^{-1} \nabla \psi_{n} A^{-1}$ converges to Id a.e. and $\operatorname{det} \nabla \psi_{n}$ converges to $(\operatorname{det} G)^{1 / 2}$ a.e. and in $L^{1}\left(O ; \mathbb{R}^{3}\right)$ for instance. By Ball's argument again, $\operatorname{det} \nabla \psi=(\operatorname{det} G)^{1 / 2}$ which excludes $\psi$ constant. 
We adopt the above modeling for a sequence of thin structures with reference configurations $\left.\Omega^{h}=\omega \times\right]-\frac{h}{2}, \frac{h}{2}[$ and with a target metric only depending on the horizontal variables $\bar{x}=\left(x_{1}, x_{2}\right)$. We consider their energies, scaled by $h$,

$$
I^{h}(\psi)=\frac{1}{h} \int_{\Omega^{h}} W\left(\bar{x}, \nabla \psi(x) A^{-1}(\bar{x})\right) \mathrm{d} x-\frac{1}{h} \int_{\Omega^{h}} f^{h}(x) \cdot \psi(x) \mathrm{d} x .
$$

By the standard change of variables $\phi(x)=\psi\left(\bar{x}, h x_{3}\right)$ and keeping the same notation $x$ for an element of $\Omega=\omega \times]-\frac{1}{2}, \frac{1}{2}[$, we rewrite the energies as

$$
I^{h}(\phi)=\int_{\Omega} W\left(\bar{x}, \nabla_{h} \phi(x) A^{-1}(\bar{x})\right) \mathrm{d} x-\int_{\Omega} f(x) \cdot \phi(x) \mathrm{d} x,
$$

where $\phi: \Omega \mapsto \mathbb{R}^{3}, \nabla_{h} \phi=\left[\partial_{1} \phi,\left|\partial_{2} \phi\right| \frac{\partial_{3} \phi}{h}\right]$ and where, for the sake of simplicity, $f^{h}\left(x^{\prime}, h x_{3}\right)=f\left(x^{\prime}, x_{3}\right)$

\subsection{The Euclidean membrane model}

The case when $G=\operatorname{Id}$ and $W$ does not depend on $\bar{x}$ was studied in [15] under the hypothesis that $W$ : $\mathbb{M}_{3,3} \mapsto \mathbb{R}$ obeys $p$-growth and $p$-coerciveness. It was then proved that obtaining the $\Gamma$-limit in $L^{p}\left(\Omega ; \mathbb{R}^{3}\right)$ of the internal energies

$$
E^{h}(\phi)=\int_{\Omega} W\left(\nabla_{h} \phi(x)\right) \mathrm{d} x
$$

required two steps: minimizing $W$ with respect to the third column, and quasiconvexifying. More precisely, defining $W_{0}: \mathbb{M}_{3,2} \mapsto \mathbb{R}$ by

$$
W_{0}(\bar{F})=\min \left\{W([\bar{F} \mid b]), b \in \mathbb{R}^{3}\right\},
$$

the sequence $E^{h} \Gamma$-converges in $L^{p}\left(\Omega ; \mathbb{R}^{3}\right)$ towards $E_{0}$ given by

$$
E_{0}(\phi)=\left\{\begin{array}{l}
\int_{\omega} Q W_{0}(\bar{\nabla} \varphi(\bar{x})) \mathrm{d} \bar{x}, \phi=\varphi \in W^{1, p}\left(\omega ; \mathbb{R}^{3}\right) \\
+\infty, \phi \in L^{p}\left(\Omega ; \mathbb{R}^{3}\right) \backslash W^{1, p}\left(\omega ; \mathbb{R}^{3}\right)
\end{array}\right.
$$

where $\bar{\nabla} \varphi(\bar{x})=\left(\partial_{1} \varphi(\bar{x}) \mid \partial_{2} \varphi(\bar{x})\right)$. The degeneracy of $Q W_{0}$ under compression was also observed: $Q W_{0}$ vanishes on matrices with singular values smaller than 1 . This degeneracy is a consequence of frame-indifference and was obtained by the explicit construction of sequence of deformations with zero elastic energy weakly converging to compressions.

\subsection{The prestrained membrane model}

We now turn to energies involving a target metric $G$. Keeping for simplicity the same notation, we define

$$
E^{h}(\phi)=\int_{\Omega} W\left(\bar{x}, \nabla_{h} \phi(x) A^{-1}(\bar{x})\right) \mathrm{d} x
$$

on $W^{1, p}\left(\Omega ; \mathbb{R}^{3}\right)$. It is an easy matter to extend the reasonings of [15] and to prove that $E^{h} \Gamma$-converges in $L^{p}\left(\Omega ; \mathbb{R}^{3}\right)$ towards $E_{0}$ defined by

$$
E_{0}(\phi)=\left\{\begin{array}{l}
\int_{\omega} Q W_{0}^{A}(\bar{x}, \bar{\nabla} \varphi(\bar{x})) \mathrm{d} \bar{x}, \phi=\varphi \in W^{1, p}\left(\omega ; \mathbb{R}^{3}\right) \\
+\infty, \phi \in L^{p}\left(\Omega ; \mathbb{R}^{3}\right) \backslash W^{1, p}\left(\omega ; \mathbb{R}^{3}\right)
\end{array}\right.
$$

where $W_{0}^{A}: \omega \times \mathbb{M}_{3,2} \mapsto \mathbb{R}$ is now defined by

$$
W_{0}^{A}(\bar{x}, \bar{F})=\min \left\{W\left(\bar{x},[\bar{F} \mid b] A^{-1}(\bar{x})\right), b \in \mathbb{R}^{3}\right\}
$$


The energy $E_{0}$ is a generalized membrane energy. The limit model associated with (6) is

$$
\min I_{0}(\varphi):=E_{0}(\varphi)-\int_{\omega} \int_{-\frac{1}{2}}^{\frac{1}{2}} f\left(\cdot, x_{3}\right) \cdot \varphi \mathrm{d} \bar{x} \text { on } W^{1, p}\left(\omega ; \mathbb{R}^{3}\right)
$$

with possible boundary conditions the influence of which is not our purpose here. On the contrary, suppose there is no boundary condition, as is the case on experiments on polymer films with differential growth [8], or in growing leaves, and no exterior loads (or that they are of a smaller order of magnitude), then the problem reduces to $\min E_{0}(\varphi)$ on $W^{1, p}\left(\omega ; \mathbb{R}^{3}\right)$. If this minimum is zero, and specially if it is attained on several deformations, additional information has to be sought by trying to determine the order of magnitude of $E^{h}$ when $h$ goes to 0 .

We therefore concentrate on identifying the deformations such that $E_{0}(\varphi)=0$, and first on finding the zero set of $W_{0}^{A}(\bar{x}, \cdot)$. For any matrix $M$ in $\mathbb{M}_{3,3}$, we denote by $M_{2 \times 2}$ its first principal $2 \times 2$ minor.

Lemma 1.2. For any $\bar{x} \in \omega$ and any $\bar{F}=\left[f_{1} \mid f_{2}\right]$ such that $\bar{F}^{T} \bar{F}=G_{2 \times 2}(\bar{x})$, there holds $W_{0}^{A}(\bar{x}, \bar{F})=0$. The unique vector $b$ such that $[\bar{F} \mid b] A^{-1}(\bar{x}) \in S O(3)$ is given by

$$
b=\bar{F}\left(G_{2 \times 2}\right)^{-1} \bar{g}+\left(\frac{\operatorname{det} G}{\operatorname{det} G_{2 \times 2}}\right)^{1 / 2} \frac{f_{1} \wedge f_{2}}{\left|f_{1} \wedge f_{2}\right|}=-\left(g^{33}\right)^{-1}\left(g^{13} f_{1}+g^{23} f_{2}\right)+\left(g^{33}\right)^{-(1 / 2)} \frac{f_{1} \wedge f_{2}}{\left|f_{1} \wedge f_{2}\right|}
$$

where $\bar{g}=\left(g_{13}, g_{23}\right)^{T}$ and $G^{-1}=\left(g^{i j}\right), i, j=1,2,3$.

Proof. (i) A short existence and uniqueness proof can be obtained as follows. Let $\bar{x}$ be given. Since $W(\bar{x}, \cdot)$ vanishes on $\mathrm{SO}(3), W_{0}^{A}(\bar{x}, \bar{F})=0$ for any $\bar{F} \in \mathbb{M}_{3,2}$ such that there exists $b \in \mathbb{R}^{3},[\bar{F} \mid b] A^{-1}(\bar{x}) \in \mathrm{SO}(3)$. Let us use the extended polar decomposition of $\bar{F}$, see [16],

$$
\bar{F}=R J \bar{U}, R \in \operatorname{SO}(3), J=\left(\begin{array}{ll}
1 & 0 \\
0 & 1 \\
0 & 0
\end{array}\right), \bar{U}=\left(\bar{F}^{T} \bar{F}\right)^{1 / 2}
$$

Replacing $b$ by $b=R b^{\prime}$ and writing $b^{\prime T}=\left(\bar{b}^{\prime T}, b_{3}^{\prime}\right)$ with $\bar{b}^{\prime} \in \mathbb{R}^{2}$, the condition on $\bar{F}$ is equivalent to $\left[J \bar{U} \mid b^{\prime}\right] A^{-1}(\bar{x}) \in \mathrm{SO}(3)$, that is

$$
\left[J \bar{U} \mid b^{\prime}\right]^{T}\left[J \bar{U} \mid b^{\prime}\right]=G(\bar{x}), \operatorname{det}\left[J \bar{U} \mid b^{\prime}\right]>0, \quad \text { or, } \quad\left[\begin{array}{cc}
\bar{F}^{T} \bar{F} & \bar{U} \bar{b}^{\prime} \\
\bar{b}^{\prime T} \bar{U} & b^{\prime T} b^{\prime}
\end{array}\right]=G(\bar{x}), \operatorname{det}\left[J \bar{U} \mid b^{\prime}\right]>0,
$$

where we used $\bar{U}^{2}=\bar{F}^{T} \bar{F}, J^{T} J=\operatorname{Id}_{2}$ and $J^{T} b^{\prime}=\bar{b}^{\prime}$. This imposes $\bar{F}^{T} \bar{F}=G_{2 \times 2}(\bar{x})$. Then $\bar{U} \bar{b}^{\prime}=G_{2 \times 2}^{1 / 2}(\bar{x}) \bar{b}^{\prime}$ and the remaining conditions read, at point $\bar{x}$,

$$
\bar{b}^{\prime}=\left(G_{2 \times 2}\right)^{-(1 / 2)} \bar{g}, \quad\left|\bar{b}^{\prime}\right|^{2}+b_{3}^{\prime 2}=g_{33}, \operatorname{det}\left[\begin{array}{cc}
\bar{U} & \bar{b}^{\prime} \\
0 & b_{3}^{\prime}
\end{array}\right]>0
$$

where $\bar{g}=\left(g_{13}, g_{23}\right)^{T}$. The vector $\bar{b}^{\prime}$ is defined by the first equality. The third condition is satisfied as soon as $b_{3}^{\prime}>0$. It remains to check that $\left|\bar{b}^{\prime}\right|^{2}=\left|G_{2 \times 2}^{-(1 / 2)} \bar{g}\right|^{2}<g_{33}$. This is an easy consequence of det $G>0$ since, by multiplying $G$ by $\operatorname{diag}\left(G_{2 \times 2}^{-1}, 1\right)$, one can check that $\operatorname{det} G=\left(\operatorname{det} G_{2 \times 2}\right)\left(g_{33}-\bar{g}^{T} G_{2 \times 2}^{-1} \bar{g}\right)$. Actually, $b_{3}^{\prime}=\left(\frac{\operatorname{det} G}{\operatorname{det} G_{2 \times 2}}\right)^{1 / 2}$.

(ii) Let us now turn to explicit formulas. Let $\bar{F}$ be such that $\bar{F}^{T} \bar{F}=G_{2 \times 2}(\bar{x})$. The condition $[\bar{F} \mid b] A^{-1}(\bar{x}) \in$ $\mathrm{SO}(3)$ is, omitting $\bar{x}$, equivalent to $\bar{F}^{T} b=\bar{g},|b|^{2}=g_{33}$ and $\operatorname{det}[\bar{F} \mid b]>0$. The first condition $\operatorname{defines}$ the inner product of $b$ with the two independent columns $f_{1}, f_{2}$ of $\bar{F}$ : writing $b=\lambda_{1} f_{1}+\lambda_{2} f_{2}+\lambda_{3} f_{1} \wedge f_{2}$, it reads $\lambda_{1} \bar{F}^{T} f_{1}+\lambda_{2} \bar{F}^{T} f_{2}=\bar{g}$, or else, $\left(\bar{F}^{T} \bar{F}\right) \bar{\lambda}=\bar{g}$ where $\bar{\lambda}=\left(\lambda_{1}, \lambda_{2}\right)$. Then, $b=\bar{F} \bar{\lambda}+\lambda_{3} f_{1} \wedge f_{2}=$ 
$\bar{F}\left(G_{2 \times 2}\right)^{-1} \bar{g}+\lambda_{3} f_{1} \wedge f_{2}$ and

$$
|b|^{2}=\left|G_{2 \times 2}^{-1 / 2} \bar{g}\right|^{2}+\lambda_{3}^{2} \operatorname{det} G_{2 \times 2}=g_{33}-\frac{\operatorname{det} G}{\operatorname{det} G_{2 \times 2}}+\lambda_{3}^{2} \operatorname{det} G_{2 \times 2} .
$$

Condition $|b|^{2}=g_{33}$ provides $\lambda_{3}^{2}=\frac{\operatorname{det} G}{\left(\operatorname{det} G_{2 \times 2}\right)^{2}}$. Finally, $\operatorname{det}[\bar{F} \mid b]=\lambda_{3} \operatorname{det}\left[f_{1}\left|f_{2}\right| f_{1} \wedge f_{2}\right]>0$ by choosing $\lambda_{3}>0$.

To summarize, we proved that

$$
b=\bar{F}\left(G_{2 \times 2}\right)^{-1} \bar{g}+\left(\frac{\operatorname{det} G}{\operatorname{det} G_{2 \times 2}}\right)^{1 / 2} \frac{f_{1} \wedge f_{2}}{\left|f_{1} \wedge f_{2}\right|} .
$$

We may give an alternative expression. Indeed, $g^{33}=(\operatorname{det} G)^{-1} \operatorname{det} G_{2 \times 2}$ and by block product of $G$ and $G^{-1}$,

$$
G_{2 \times 2}\left(\begin{array}{c}
g^{13} \\
g^{23}
\end{array}\right)+g^{33} \bar{g}=0
$$

which proves that $\bar{\lambda}=-\left(\frac{g^{13}}{g^{33}}, \frac{g^{23}}{g^{33}}\right)$. The vector $b$ reads equivalently

$$
b=-\left(g^{33}\right)^{-1}\left(g^{13} f_{1}+g^{23} f_{2}\right)+\left(g^{33}\right)^{-(1 / 2)} \frac{f_{1} \wedge f_{2}}{\left|f_{1} \wedge f_{2}\right|},
$$

which ends the proof.

Formulas analogous to (14) were first given in [3], [19]. We now turn to the zero set of $Q W_{0}^{A}(\bar{x}, \cdot)$. Obviously, $Q W_{0}^{A}(\bar{x}, \cdot)$ inherits the frame-indifference property of $W(\bar{x}, \cdot): Q W_{0}^{A}(\bar{x}, R \bar{F})=Q W_{0}^{A}(\bar{x}, \bar{F})$ for any $\bar{x} \in \omega$, $\bar{F} \in \mathbb{M}_{3,2}, R \in \mathrm{SO}(3)$ which allows to write $Q W_{0}(\bar{x}, \cdot)$ as a function of $\bar{C}=\bar{F}^{T} \bar{F}$. Denoting $\operatorname{Sym}_{2}^{+}$the set of positive semi-definite symmetric matrices, a consequence of Pipkin's property is that the following inequality holds

$$
Q W_{0}^{A}(\bar{x}, \bar{F}) \leq \inf \left\{Q \tilde{W}_{0}^{A}\left(\bar{x}, \bar{F}^{T} \bar{F}+S\right) ; S \in \operatorname{Sym}_{2}^{+}\right\} .
$$

Indeed, for any $Y: \mathbb{M}_{3,2} \mapsto \mathbb{R}, \mathrm{SO}(3)$ left-invariant, rank-1 convex, one has $\tilde{Y}(C) \leq \tilde{Y}(C+S)$ for any $S \in \operatorname{Sym}_{2}^{+}$. Such functions are said to be increasing. This result, see [22], and its extension to energies defined on $\mathbb{M}_{3,3}$ that are $\mathrm{O}(3)$ left-invariant, see [17], are obtained by associating with $\tilde{Y}$ functions $h$ of the real variable $t$. Those functions are convex, symmetric with respect to some $t_{0}$, hence minimum at $t_{0}$ and monotone increasing on $\left[t_{0},+\infty\right]$. From (15), we derive that $Q W_{0}^{A}(\bar{x}, \bar{F})=0$ for any $\bar{F}$ such that $\bar{F}^{T} \bar{F} \leq G_{2 \times 2}(\bar{x})$. We immediately obtain the following result on deformations.

Proposition 1.3. The membrane energy $E_{0}$ vanishes on deformations $\varphi \in W^{1, p}\left(\omega ; \mathbb{R}^{3}\right)$ such that $(\nabla \varphi)^{T} \nabla \varphi \leq$ $G_{2 \times 2}$, that are the short maps of $G_{2 \times 2}$.

We emphasize the fact that Proposition 1.3 has been obtained as a consequence of the algebraic property of $Q W_{0}^{A}$. It is one of the rare cases when a result on quasiconvex functions or on weakly lower semi-continuous energies acting on $W^{1, p}$ can be obtained by algebraic reasoning.

At this point, we are facing a key feature of the mechanical process: If the Riemannian curvature tensor of $G$ is not zero, then the $3 \mathrm{~d}$ systems cannot reach the metric (and their energetical infima are strictly positive). Nevertheless, inf $E^{h}$ converges to 0 and the limit model has many solutions, that are deformations from $\mathbb{R}^{2}$ into $\mathbb{R}^{3}$ with zero membrane energy. Indeed, the existence of an ample set of $\mathcal{C}^{1}$ isometric immersions of $G_{2 \times 2}$ from $\omega \subset \mathbb{R}^{2}$ into $\mathbb{R}^{3}$ was obtained by Nash and Kuiper in celebrated papers. It is of importance to notice that the regularity they asked was not $\mathcal{C}^{2}$ in which case the result is lost. Recent papers [5], [7] improved the regularity to $\mathcal{C}^{1, \alpha}, \alpha<\frac{1}{7}$ and still better $\alpha<\frac{1}{5}$. In the meantime explicit visualization based on Gromov convex integration method was developed in the Hevea project, see http://hevea.imag.fr/ for beautiful representations. 
The next natural question is to try and find the true order of magnitude of the energies $E^{h}$ in terms of $h$ since we have seen that it is not $\mathcal{O}(1)$. This question is still under debate in the Euclidean case $(A=\mathrm{Id})$ : for $q<\frac{5}{3}, \frac{E^{h}}{h^{q}} \Gamma$-converges to the functional which is 0 on short maps, $+\infty$ otherwise, see [6]. The same reference gives partial results for $q=\frac{5}{3}$. No result is known for $\frac{5}{3} \leq q<2$. For $q=2, A=\mathrm{Id}$, it is well known that the limit model is the bending model, see [9] for the first heuristic derivation, [10] for a variational proof based on the so-called quantitative rigidity estimate. This estimate, proved in [10], [11], consists in a quantitative version of Liouville theorem which states that, for a connected open subset $O$ of $\mathbb{R}^{3}$, any $\psi$ in $W^{1,1}\left(O ; \mathbb{R}^{3}\right)$ such that $\nabla \psi^{T} \nabla \psi=\mathrm{Id}$, det $\nabla \psi(x)>0$, is a rotation. The quantitative estimate says that, for any $\psi$ in $H^{1}\left(O ; \mathbb{R}^{3}\right)$,

$$
\exists R \in \mathrm{SO}(3) \text { independent of } x,\|\nabla \psi-R\|_{L^{2}\left(O ; \mathbb{M}_{3,3}\right)} \leq C(O)\|\operatorname{dist}(\nabla \psi, \mathrm{SO}(3))\|_{L^{2}(O)} .
$$

Moreover, the dependency of the constant $C$ on slender domains $\left.\Omega^{h}=\omega \times\right]-\frac{h}{2}, \frac{h}{2}[, h$ going to 0 , is provided. Written alternatively on $\Omega=\omega \times]-\frac{1}{2}, \frac{1}{2}$ [ with the $h$-dependent gradient, it reads:

$$
\exists c(\omega)>0, \forall \phi \in H^{1}\left(\Omega ; \mathbb{R}^{3}\right), \forall h, \exists R: \omega \mapsto \mathrm{SO}(3),\left\{\begin{array}{l}
\left\|\nabla_{h} \phi-R\right\|_{L^{2}\left(\Omega ; \mathbb{M}_{3,3}\right)} \leq c(\omega)\left\|\operatorname{dist}\left(\nabla_{h} \phi, \operatorname{SO}(3)\right)\right\|_{L^{2}(\Omega)}, \\
\|\bar{\nabla} R\|_{L^{2}\left(\omega ;\left(\mathbb{M}_{3,3}\right)^{2}\right)} \leq \frac{c(\omega)}{h}\left\|\operatorname{dist}\left(\nabla_{h} \phi, \operatorname{SO}(3)\right)\right\|_{L^{2}(\Omega)},
\end{array}\right.
$$

(when $\operatorname{dist}\left(\nabla_{h} \phi, \mathrm{SO}(3)\right)$ is already of order $h^{2}$ which is the relevant case). In the next section, we review results which show that for arbitrary imposed metrics, models of order 2 may be not possible. Indeed, they can be obtained only if $G_{2 \times 2}$ admits regular enough isometric immersions.

\section{BENDING MODEL}

\subsection{The Euclidean bending model}

Let us first recall results in the classical case $G=\mathrm{Id}$. The energy of the membrane model seen in the previous section is expressed in terms of $Q W_{0}$, where $W_{0}$ is the mimimum of $W$ with respect to the third column as in (8). Minimizers make $Q W_{0}$ equal to 0 . The bending model uses the second derivative of $W$, which can be expected since $W^{\prime}(R)=0$ for any $R \in \mathrm{SO}(3)$. It is easily seen that as a consequence of frame indifference

$$
W^{\prime \prime}(\operatorname{Id})(F, F)=W^{\prime \prime}(\mathrm{Id})(\operatorname{sym} F, \operatorname{sym} F) .
$$

Indeed, let $f(t)=W(\operatorname{Id}+t F)=W(D(t))$ where $D(t)=\left((\operatorname{Id}+t F)^{T}(\operatorname{Id}+t F)\right)^{1 / 2}$. Differentiating $D^{2}(t)$, we obtain $D^{\prime}(0)=\operatorname{sym} F$, then $W^{\prime \prime}(\operatorname{Id})(F, F)=f^{\prime \prime}(0)=W^{\prime \prime}(\operatorname{Id})\left(D^{\prime}(0), D^{\prime}(0)\right)=W^{\prime \prime}(\operatorname{Id})(\operatorname{sym} F, \operatorname{sym} F)$.

Under the assumption that $W(F) \geq C \operatorname{dist}^{2}(F, \mathrm{SO}(3))$, a first finding, [10], is that any sequence $\phi^{h}$ whose energy satisfies $E^{h}\left(\phi^{h}\right) \leq C h^{2}$ admits a subsequence such that $\nabla_{h} \phi^{h}$ converges to $(\bar{\nabla} \varphi, b)$ in $L^{2}(\Omega)$ with $(\bar{\nabla} \varphi, b) \in \mathrm{SO}(3)$, and with the additional regularities $\varphi \in H^{2}(\omega), b \in H^{1}(\omega)$. For $F^{\sharp} \in \mathbb{M}_{2}$, let

$$
\begin{aligned}
W_{2}\left(F^{\sharp}\right) & =\min \left\{W^{\prime \prime}(\mathrm{Id})(F, F) ; F \in \mathbb{M}_{3,3}, F_{2 \times 2}=F^{\sharp}\right\} \\
& =\min \left\{W^{\prime \prime}(\mathrm{Id})(\operatorname{sym} F, \operatorname{sym} F) ; F \in \mathbb{M}_{3,3}, F_{2 \times 2}=F^{\sharp}\right\} .
\end{aligned}
$$

Then, [10], the following $\Gamma$-convergence result holds:

$$
\frac{E^{h}}{h^{2}} \stackrel{\Gamma-H^{1}\left(\Omega ; \mathbb{R}^{3}\right)}{\longrightarrow} E_{2}, E_{2}(\phi)=\left\{\begin{array}{l}
\frac{1}{4 !} \int_{\omega} W_{2}\left(\left(\bar{\nabla} \varphi^{T} \bar{\nabla} n\right)(\bar{x})\right) \mathrm{d} \bar{x}, \phi=\varphi \in H^{2}\left(\omega ; \mathbb{R}^{3}\right), \text { isometry, } \\
+\infty \text { otherwise }
\end{array}\right.
$$

where $n=\frac{\partial_{1} \varphi \wedge \partial_{1} \varphi}{\left|\partial_{1} \varphi \wedge \partial_{1} \varphi\right|}$ is the unit normal to the deformed surface $\varphi(\omega)$ and $\mathcal{B}(\varphi)=\bar{\nabla} \varphi^{T} \bar{\nabla} n$ is the second fundamental form of $\varphi(\omega)$, symmetric by definition. The abstract $\Gamma$-convergence result makes sense since obviously there are deformations such that $E^{h}\left(\phi^{h}\right) \leq C h^{2}$. For a Saint Venant-Kirchhoff material or, more 
generally, for an isotropic material, one recovers the well-known energy density $\frac{1}{4 !}\left(2 \mu|\mathcal{B}(\varphi)|^{2}+\frac{2 \mu}{2 \mu+\lambda}|\operatorname{tr} \mathcal{B}(\varphi)|^{2}\right)$. The limit energy $E_{2}(\phi)$ is finite on $H^{2}(\omega)$-isometries, that is mappings from $\omega$ into $\mathbb{R}^{3}$ whose first fundamental form is $\operatorname{Id}_{2}$. The energy depends on their second fundamental form that measures the curvature (or bending). It vanishes on isometries whose curvature is zero: $\varphi=R(\bar{x}, 0)+c, R \in \mathrm{SO}(3)$.

\subsection{The prestrained bending model}

An essential difference between the Euclidean case and the case with imposed metric occurs at this stage. Indeed, as shown in [18], [3], and as will be stated below, $\frac{E^{h}}{h^{2}}$ may have a $\Gamma$-limit taking some finite values only if $G_{2 \times 2}$ admits an isometry with second derivatives in $L^{2}(\omega)$. In Section 2.1, we already noticed that the subset on which $E_{2}$ is meaningful was the subset of $\mathrm{H}^{2}$-regular isometries, whose examples in the Euclidean case are numerous, the most obvious ones being deformations into planes, cylinders, cones. This gives information on what $\varphi$ can be and, at the same time, shows that the $h^{2}$ order of magnitude is relevant.

For an arbitrary $G_{2 \times 2}$, the situation is drastically different. Indeed, as mentioned in Section 1.2, finding a $\mathcal{C}^{1, \alpha}$ isometry, $\alpha<\frac{1}{5}$, is always possible, but second order derivatives cannot always be defined as functions. For simplicity, we drop the first argument in $W$ and we are concerned with internal energies $W\left(\nabla_{h} \phi(x) A^{-1}(\bar{x})\right)$. The extension of Section 2.1 works as follows, see [18], [3]. For $F^{\sharp} \in \mathbb{M}_{2,2}$, let

$$
W_{2}^{A}\left(\bar{x}, F^{\sharp}\right)=\min \left\{W^{\prime \prime}(\mathrm{Id})\left(A^{-1}(\bar{x}) F A^{-1}(\bar{x})\right)^{(2)} ; F \in \mathbb{M}_{3,3}, F_{2 \times 2}=F^{\sharp}\right\} .
$$

Note that again $W_{2}^{A}\left(\bar{x}, F^{\sharp}\right)$ only depends on $\operatorname{sym} F^{\sharp}$. It is proved in [18], [3] that inf $\frac{E^{h}}{h^{2}} \leq C$ if and only if $G_{2 \times 2}$ admits a $H^{2}\left(\omega ; \mathbb{R}^{3}\right)$ isometry. Then, the variational convergence result of Section 2.1 extends in

$$
\frac{E^{h}}{h^{2}} \stackrel{\Gamma-H^{1}\left(\Omega ; \mathbb{R}^{3}\right)}{\longrightarrow} E_{2}, E_{2}(\phi)=\left\{\begin{array}{l}
\frac{1}{4 !} \int_{\omega} W_{2}^{A}\left(\bar{x},\left(\bar{\nabla} \varphi^{T} \bar{\nabla} b\right)(\bar{x})\right) d \bar{x}, \phi=\varphi \in H^{2}\left(\omega ; \mathbb{R}^{3}\right), G_{2 \times 2} \text {-isometry } \\
+\infty \text { otherwise }
\end{array}\right.
$$

where $b \in\left(H^{1} \cap L^{\infty}\right)\left(\omega ; \mathbb{R}^{3}\right)$ is uniquely defined in terms of $\varphi$ (that already solves $\nabla \varphi^{T} \bar{\nabla} \varphi=G_{2 \times 2}$ ) as at the membrane level, i.e.,

$$
\left(\begin{array}{cc}
\bar{\nabla} \varphi^{T} \bar{\nabla} \varphi & \partial_{\alpha} \varphi \cdot b \\
\partial_{\alpha} \varphi \cdot b & |b|^{2}
\end{array}\right)=G=\left(\begin{array}{cc}
{\left[g_{\alpha \beta}\right]} & g_{\alpha 3} \\
g_{\alpha 3} & g_{33}
\end{array}\right), \operatorname{det}\left[\partial_{1} \varphi\left|\partial_{2} \varphi\right| b\right]>0
$$

It is easily seen that, if for instance $g_{\alpha 3}=0, \alpha=1,2$, then $b=\left(g^{33}\right)^{-(1 / 2)} n$, where $n=\frac{\partial_{1} \varphi \wedge \partial_{2} \varphi}{\left|\partial_{1} \varphi \wedge \partial_{2} \varphi\right|}$ is the unit normal to $\varphi(\omega)$ oriented by $\varphi$. More generally, from (14),

$$
b=-\left(g^{33}\right)^{-1}\left(g^{13} \partial_{1} \varphi+g^{23} \partial_{2} \varphi\right)+\left(g^{33}\right)^{-(1 / 2)} n .
$$

If $G_{2 \times 2}$ has no $H^{2}$-isometric immersion, then the imposed metric is a blocking mechanism that obliges the energies to remain larger than $\mathcal{O}\left(h^{2}\right)$. In the case when there is a $H^{2}$-isometric immersion, the next question is to determine whether the minimum of $E_{2}$ can be zero or not. By (19),

$$
\min E_{2}=0 \Leftrightarrow \exists \varphi \in H^{2}\left(\omega ; \mathbb{R}^{3}\right), \bar{\nabla} \varphi(\bar{x})^{T} \bar{\nabla} \varphi(\bar{x})=G_{2 \times 2}, \bar{\nabla} \varphi^{T} \bar{\nabla} b \text { is skew-symmetric. }
$$

We check again that for $G$ block-diagonal the skew-symmetry condition means that $\mathcal{B}(\varphi)=0$. In the general case, using expression (20), we can compute $\bar{\nabla} \varphi^{T} \bar{\nabla} b$. Terms arising from the components of $b$ along $\partial_{1} \varphi$ and $\partial_{2} \varphi$ are linear combinations of $\partial_{\alpha} \varphi \cdot \partial_{\beta} \varphi$ and $\partial_{\alpha} \varphi \cdot \partial_{\alpha \beta} \varphi, \alpha, \beta=1,2$, which are known in terms of $G_{2 \times 2}$ since $\varphi$ satisfies the isometry condition. Since $\partial_{\alpha} \varphi \cdot n=0$, terms arising from the component of $b$ along $n$ reduce to $\left(g^{33}\right)^{-(1 / 2)} \partial_{\alpha} \varphi \cdot \partial_{\beta} n$. As $\left(g^{33}\right)^{-(1 / 2)} \neq 0$, writing that $\bar{\nabla} \varphi^{T} \bar{\nabla} b$ is skew-symmetric provides an explicit formula for the second fundamental form $\bar{\nabla} \varphi^{T} \bar{\nabla} n$. As a consequence, when there exists $\varphi$ such that (21) holds, it is 
unique up to a rigid transformation. Now, it is of interest to characterize the metrics such that (21) can actually occur. The result is amazingly concise and its proof can be found in [3].

Proposition 2.1. A metric $G(\bar{x})$ is such that $\min E_{2}=0$, or, alternatively, is such that $G_{2 \times 2}$ admits a $H^{2}$-regular isometric immersion $\varphi$ with $\bar{\nabla} \varphi^{T} \bar{\nabla} b$ skew-symmetric, if and only if the Riemann curvatures $R_{1212}, R_{1213}, R_{1223}$ of $G$ are equal to 0 (which is equivalent to $R_{\alpha \beta i j}=0$ for $\alpha, \beta=1,2, i, j=1,2,3$ ).

Proof. See [3]. The proof consists of two steps. First, it is shown that for a $H^{2}$-regular isometric immersion $\varphi$ of $G_{2 \times 2}$ the fact that $\bar{\nabla} \varphi^{T} \bar{\nabla} b$ is skew-symmetric is equivalent to $\mathcal{B}_{\alpha \beta}(\varphi)=-\left(g^{33}\right)^{-(1 / 2)} \Gamma_{\alpha \beta}^{3}, \alpha, \beta=1,2$, where $\Gamma_{i j}^{p}, i, j, p=1,2,3$ are the Christoffel symbols of the second kind of $G$. Then, appropriate calculations show that the Codazzi-Mainardi and Gauss equations that connect $G_{2 \times 2}$ and $\mathcal{B}(\varphi)$ reduce to the vanishing of $R_{1212}, R_{1213}, R_{1223}$. When this condition is satisfied, the converse property implies that there is a smooth, hence $H^{2}$, mapping $\varphi$ with the prescribed fundamental forms.

\section{Generalized von KÁrmán model}

From now on, we are interested in the deformation behaviors when $R_{12 i j}=0, i, j=1,2,3$, so that the minimum of the energy of the order 2 model of Section 2 is 0 and that the infima of $\frac{E^{h}}{h^{2}}$ converge to 0 . Again, the question of their true order of magnitude arises.

\subsection{The Euclidean von Kármán model}

A formal derivation of the von Kármán model was given in [4] by means of asymptotic expansions both on the displacements and on the stresses for an appropriate order of magnitude of the loads (roughly speaking, horizontal components of order 2 and vertical component of order 3) and an appropriate guess of the orders of magnitude of the separate displacement and stress components. This allowed to see the von Kármán model as a generalization of the linearized elasticity model that at that time was rigorously justified by convergence results, to the extent that it was derived from the $3 \mathrm{~d}$ linearized system of elasticity. An important contribution from [9] was to insert the von Kármán model in a hierarchy of models of thin structures, to show that its place is below the membrane and the bending model, and actually above the linear model. This was made rigorous by the variational convergence results in [11]. The von Kármán model is still a nonlinear model, but the higher order derivatives appear linearly. It is valid close to the identity, in other words in the small displacement regime. It expresses in terms of the leading order terms $u_{1}^{2}, u_{2}^{2}$ of the horizontal components of the displacement vector and of the leading order term $u_{3}^{1}$ of the vertical component. Its internal energy reads

$$
E_{4}\left(u_{1}^{2}, u_{2}^{2}, u_{3}^{1}\right)=\frac{1}{2} \int_{\omega} W_{2}\left(\left[\frac{\partial_{\alpha} u_{\beta}^{2}+\partial_{\beta} u_{\alpha}^{2}+\partial_{\alpha} u_{3}^{1} \partial_{\beta} u_{3}^{1}}{2}\right]\right) \mathrm{d} \bar{x}+\frac{1}{4 !} \int_{\omega} W_{2}\left(\left[\partial_{\alpha \beta} u_{3}^{1}\right]\right) \mathrm{d} \bar{x}
$$

where the indices in the brackets run over $1,2, W_{2}$ has been defined in (17) and is quadratic in its argument. The energy $E_{4}$ consists of the sum of a stretching term and of a bending term.

\subsection{The prestrained von Kármán model}

As in the previous sections, we now come back to our main topic, namely imposed metrics, where an extremely nice feature can be proved: as soon as the internal energy $E_{2}$ can be minimized to 0 , then, automatically, inf $E^{h}$ is of order $h^{4}$ or is smaller. The following lemma is proved in [19].

Lemma 3.1. Let $G(\bar{x})$ be a metric such that $R_{12 i j}=0, i, j=1,2,3$, then inf $E^{h} \leq C h^{4}$.

Let us give a hint of the proof so that the reader can see how a new vector-field $d$ comes into play. 
Proof. It suffices to exhibit a sequence of mappings $\phi^{h}$ whose energies are of the order of or smaller than $h^{4}$. Quite naturally, we use the information given by the order 0 and order 2 models on the form of the leading term of the deformations and try and go a step further. Let $\phi^{h}$ be defined by $\phi^{h}\left(\bar{x}, x_{3}\right)=\varphi(\bar{x})+h x_{3} b(\bar{x})+h^{2} \frac{x_{3}^{2}}{2} d(\bar{x})$, where $\varphi$ and $b$ are uniquely determined as in Section 2 and $d$ will make terms candidate to be of higher order in the energies disappear. Indeed, $\nabla_{h} \phi^{h} A^{-1}\left(\bar{x}, x_{3}\right)=\left(Q A^{-1}\right)\left(\operatorname{Id}+h x_{3} A^{-1} Q^{T} B A^{-1}+h^{2} x_{3}^{2} T\right)$ with

$$
Q=\left[\partial_{1} \varphi\left|\partial_{2} \varphi\right| b\right], B=\left[\partial_{1} b\left|\partial_{2} b\right| d\right], D=\left[\partial_{1} d\left|\partial_{2} d\right| 0\right]
$$

and $T$ an appropriate matrix. Since $Q A^{-1} \in \mathrm{SO}(3)$,

$$
W\left(\nabla_{h} \phi^{h} A^{-1}\right)=W\left(\mathrm{Id}+h x_{3} A^{-1} Q^{T} B A^{-1}+h^{2} x_{3}^{2} T\right) .
$$

Let us check that we can choose $d$ such that $Q^{T} B$ is skew-symmetric which will cancel the $h^{2}$ term that otherwise would be present as a coefficient of $W^{\prime \prime}(\mathrm{Id})$. Actually,

$$
Q^{T} B=\left(\begin{array}{cc}
\bar{\nabla} \varphi^{T} \bar{\nabla} b & \bar{\nabla} \varphi^{T} d \\
b^{T} \bar{\nabla} b & b \cdot d
\end{array}\right)
$$

and since we already know that the left-upper block is skew-symmetric, it suffices to define $d$ by its inner product with $\partial_{\alpha} \varphi, \alpha=1,2$ and $b$ to make the $3 \times 3$ matrix above skew-symmetric. More precisely,

$$
Q^{T} d=\left(-b \cdot \partial_{1} b\left|-b \cdot \partial_{2} b\right| 0\right)^{T}
$$

defines $d$.

Proving $\Gamma$-convergence towards the prestrained von Kármán energy requires much more elaborate proofs than the hint given above. We refer to [19] for the analytic tools and for the computation of the several terms the limit energy consists of. We will simply say that we have to prove some compactness for sequences $\phi^{h}$ such that $E^{h}\left(\phi^{h}\right) \leq C h^{4}$. We first prove that their gradients are close to $Q(\bar{x})+h x_{3} B(\bar{x})$ up to $\bar{x}$-dependent rotations and that the gradients of the rotations are controlled as follows:

$$
\int_{\Omega}\left|\nabla_{h} \phi^{h}(x)-R^{h}(\bar{x})\left(Q(\bar{x})+h x_{3} B(\bar{x})\right)\right|^{2} \mathrm{~d} x \leq C h^{4}, \quad \int_{\omega}\left|\bar{\nabla} R^{h}(\bar{x})\right|^{2} \mathrm{~d} \bar{x} \leq C h^{2} .
$$

Then, we prove existence of constants rotations $R^{h}$ and vectors $c^{h}$ such that $y^{h}=R^{h} \phi^{h}-c^{h}$ has the properties below that, for the sake of clarity, are enunciated on $\phi^{h}$. At this stage, convergence results of $\phi^{h}$ towards $\varphi$ and of $\frac{\partial_{3} \phi^{h}}{h}$ towards $b$ are already known. We complement them with the model that the discrepancy from $\varphi+h x_{3} b$ satisfies. We recall that $W_{2}^{A}$ has been defined in (18), $Q$ in (22), and $d$ in (23).

Proposition 3.2. For any minimizing sequence $\phi^{h}$ of $E^{h}$, up to a subsequence,

- the $h^{-1}$ scaled averaged displacements $u^{h}(\bar{x}):=\frac{1}{h} \int_{-\frac{1}{2}}^{\frac{1}{2}}\left(\phi^{h}-\left(\varphi+h x_{3} b\right)\right) \mathrm{d} x_{3}$ converge in $H^{1}\left(\omega ; \mathbb{R}^{3}\right)$ to a limiting field $u^{1}$ such that $\operatorname{sym}\left((\bar{\nabla} \varphi)^{T} \bar{\nabla} u^{1}\right)=0$,

- the $h^{-2}$ scaled strains $\left(\operatorname{sym}\left((\bar{\nabla} \varphi)^{T} \frac{\bar{\nabla} u^{h}}{h}\right)\right)$ converge in $L^{2}\left(\omega ; \mathbb{M}_{2,2}\right)$ to some $\mathrm{e}^{2} \in L^{2}\left(\omega ; \mathbb{M}_{2,2}^{\mathrm{sym}}\right)$,

- any limiting pair $\left(u^{1}, \mathrm{e}^{2}\right)$ minimizes the energy

$$
\begin{aligned}
E_{4}\left(u^{1}, \mathrm{e}^{2}\right) & =\frac{1}{2} \int_{\omega} W_{2}^{A}\left(\bar{x}, \mathrm{e}^{2}+\frac{1}{2}\left(\bar{\nabla} u^{1}\right)^{T} \bar{\nabla} u^{1}+\frac{1}{4 !} \bar{\nabla} b^{T} \bar{\nabla} b\right) \mathrm{d} \bar{x} \\
& +\frac{1}{4 !} \int_{\omega} W_{2}^{A}\left(\bar{x}, \bar{\nabla} \varphi^{T} \bar{\nabla} p^{1}+\left(\bar{\nabla} u^{1}\right)^{T} \bar{\nabla} b\right) \mathrm{d} \bar{x}+\frac{1}{2 \times 6 !} \int_{\omega} W_{2}^{A}\left(\bar{x}, \operatorname{sym}\left(\bar{\nabla} \varphi^{T} \bar{\nabla} d\right)+\bar{\nabla} b^{T} \bar{\nabla} b\right) \mathrm{d} \bar{x}
\end{aligned}
$$


over the product space $\left.\left\{v \in H^{2}\left(\omega ; \mathbb{R}^{3}\right), \operatorname{sym}\left((\bar{\nabla} \varphi)^{T} \nabla v\right)=0\right\} \times \mathrm{cl}_{L^{2}}\left\{\operatorname{sym}\left((\bar{\nabla} \varphi)^{T} \nabla v\right)\right) ; v \in H^{1}\left(\omega ; \mathbb{R}^{3}\right)\right\}$. The term $p^{1}$ is defined by $u^{1}$ through $Q^{T} p^{1}=\left(-b \cdot \partial_{1} u^{1},-b \cdot \partial_{2} u^{1}, 0\right)^{T}$.

The reader familiar with the standard von Kármán model will easily recognize some expressions: $2 \mathrm{e}^{2}+$ $\left(\bar{\nabla} u^{1}\right)^{T} \bar{\nabla} u^{1}$ is a generalization of $\left[\partial_{\alpha} u_{\beta}^{2}+\partial_{\beta} u_{\alpha}^{2}+\partial_{\alpha} u_{3}^{1} \partial_{\beta} u_{3}^{1}\right]$ and $\bar{\nabla} \varphi^{T} \bar{\nabla} p^{1}$ is a generalization of $\left[-\partial_{\alpha \beta} u_{3}^{1}\right]$ since $p^{1}=-\left(\partial_{1} u_{3}^{1}, \partial_{2} u_{3}^{1}, 0\right)$ when $G=$ Id. The first two terms of $E_{4}$ are a stretching term and a bending term. The third term has an illuminating geometrical meaning in terms of the imposed metric. Indeed, as shown in [19], one can prove that

$$
\operatorname{sym}\left(\bar{\nabla} \varphi^{T} \bar{\nabla} d\right)+\bar{\nabla} b^{T} \bar{\nabla} b=\left(\begin{array}{ll}
R_{1313} & R_{1323} \\
R_{1323} & R_{2323}
\end{array}\right) .
$$

Coefficients $R_{1313}, R_{1323}, R_{2323}$ are the three entries of the Riemannian curvature tensor that were left free after we fixed the three tangential ones to 0 in this section.

As a consequence, when these three entries $R_{\alpha 3 \beta 3}, \alpha, \beta=1,2$ are not simultaneously equal to 0 , there is a positive constant term in $I_{4}$. Then, the minimum of $I_{4}$ cannot be 0 , the imposed metric does not allow the internal energy to relax. On the contrary, when all $R_{\alpha 3 \beta 3}, \alpha, \beta=1,2$, are equal to 0 , then min $E_{4}=0$, but in that case the full Riemannian tensor vanishes which means that the $3 d$-metric $G$ we started from was realizable.

We summarize by saying that as soon as the metric $G(\bar{x})$ has a $H^{2}(\omega)$-regular $G_{2 \times 2}$-isometric immersion, then three separate orders of magnitude are possible: i) inf $E^{h}=\mathcal{O}\left(h^{2}\right)$; ii) inf $E^{h}=\mathcal{O}\left(h^{4}\right)$ which corresponds to $\mathcal{R} \neq 0$, but $R_{1313}=R_{1323}=R_{2323}=0$; iii) $\mathcal{R}=0$ and $\min E^{h}=0$ for all $h$.

Work under progress in devoted to metrics that also depend on the vertical variable.

\section{REFERENCES}

[1] J.M. Ball, Convexity conditions and existence theorems in nonlinear elasticity, Arch. Rational Mech. Anal. 63 (1977), 337403.

[2] M. Ben Amar, M.M. Müller, M. Trejo, Petal shapes of sympetalous flowers: the interplay between growth, geometry and elasticity, New J. Physics 14 (2012), 085014.

[3] K. Bhattacharya, M. Lewicka and M. Schaffner, Plates with incompatible prestrain, Arch. Rational Mech. Anal. 221 (2016), 143-181.

[4] P.G. Ciarlet, P. Destuynder, A justification of a nonlinear model in plate theory, Methods Appl. Mech. Engrg. 1718 (1979), $227-258$.

[5] S. Conti, C. De Lellis, L. Székelyhidi, H-principle and rigidity for $C^{1, \alpha}$ isometric embeddings, in Nonlinear partial differential equations, The Abel Symposium 2010, H. Holden and K. H. Karlsen, Eds., Springer-Verlag (2012).

[6] S. Conti, F. Maggi, Confining thin sheets and folding paper, Arch. Ration. Mech. Anal. 187 (2008), 1-48.

[7] C. De Lellis, Inauen, L. Székelyhidi JR., A Nash-Kuiper theorem for $C^{1, \frac{1}{5}-\delta}$ immersions of surfaces in 3 dimensions, to appear in Revista matemática Iberoamericana.

[8] E. Efrati, E. Sharon, R. Kupferman, Elastic theory of unconstrained non-Euclidean plates, J. Mech. Phys. Solids 57 (2009), $762-775$.

[9] D.D. Fox, A. Raoult, J.C. Simo, A justification of nonlinear properly invariant plate theories, Arch. Rational Mech. Anal. 124 (1993), 157-199.

[10] G. Friesecke, R. James, S. Müller, A theorem on geometric rigidity and the derivation of nonlinear plate theory from three dimensional elasticity, Comm. Pure Appl. Math. 55 (2002), 1461-1506.

[11] G. Friesecke, R. James, S. MülleR, A hierarchy of plate models derived from nonlinear elasticity by Gamma-convergence, Arch. Ration. Mech. Anal. 180 (2006), 183-236.

[12] J. Gemmer and S. Venkataramani, Shape selection in non-Euclidean plates, Physica D: Nonlinear Phenomena 240 (2011), $1536-1552$.

[13] Y. Klein, E. Efrati, E. Sharon, Shaping of elastic sheets by prescription of non-Euclidean metrics, Science 315 (2007), $1116-1120$.

[14] Y. Klein, S. C. Venkataramani, E. Sharon, Experimental Study of Shape Transitions and Energy Scaling in Thin NonEuclidean Plates, Physical Rev. Letters 106 (2011).

[15] H. Le Dret, A. Raoult, The nonlinear membrane model as a variational limit of nonlinear three-dimensional elasticity, $J$. Math. Pures Appl. 73 (1995), 549-578.

[16] H. Le Dret, A. Raoult, Remarks on the quasiconvex envelope of stored energy functions, Comm. Appl. Nonlinear Anal. 1 (1994), 85-96. 
[17] H. Le Dret, A. Raoult, Quasiconvex envelopes of stored energy densities that are convex with respect to the strain tensor, in Progress in Partial Differential Equations, C. Bandle, J. Bemelmans, M. Chipot, J. Saint Jean Paulin, I Shafrir, Eds., Pitman (1995), 138-146.

[18] M. Lewicka, R. PAKzad, Scaling laws for non-Euclidean plates and the $W^{2,2}$ isometric immersions of Riemannian metrics, ESAIM: Control, Optimisation and Calculus of Variations 17 (2011), 1158-1173.

[19] M. Lewicka, A. Raoult, D. Ricciotti, Plates with incompatible prestrain of high order, Ann. Inst. H. Poincaré Anal. Non Linéaire 34 (2017), 1883-1912, 10.1016/j.anihpc.2017.01.003

[20] H. Liang, L. Mahadevan, The shape of a long leaf, Proc. Nat. Acad. Sci. (2009), 10.1073/pnas.0911954106

[21] M. Ortiz, G. Gioia, The morphology and folding patterns of buckling-driven thin-film blisters, J. Mech. Phys. Solids, 42 (1994), 531-559.

[22] A.C. Pipkin, Relaxed energy densities for large deformations of membranes, IMA J. Appl. Math 52 (1994), 297-308.

[23] J. Kim, J.A. Hanna, M. Byun, C.D. Santangelo, R.C. Hayward, Designing responsive buckled surfaces by halftone gel lithography, Science 335 (2012), 1201-1205.

[24] T.H. Ware, M.E. McConney, J.J. Wie, V.P. Tondiglia., T.J. White, Voxelated liquid crystal elastomers, ÊScience $\mathbf{3 4 7}$ (2015) 982-984. DOI:10.1126/science.1261019 